\title{
Contextual Knowledge: From Globalization to Global Aging ${ }^{1}$
}

\author{
Hongtu Chen \\ SuE LeVKofF \\ ARTHUR KLEINMAN
}

\begin{abstract}
As our general view of global history and societal development has shifted towards a more integrated approach, we face the challenge of finding the best ways to achieve integration across geographical and cultural distances. In dealing with the rising challenges associated with the global trend of demographic aging, it is argued that obtaining and accumulating contextual knowledge of local practice can be critical and productive especially in the planning phase of an eldercare intervention project. Three basic types of contextual knowledgei.e., the context of experience, context of praxis, and context of theory-which are crucial for understanding eldercare practice situations, are discussed, along with its possible impacts on not only the understanding of the eldercare situation in a specific location, but also the choice and implementation of effective intervention solutions.
\end{abstract}

Keywords: Contextual knowledge, global aging, practice

Résumé. Comme notre vue générale de l'histoire mondiale et du développement social a évolué vers une approche plus intégrée, nous sommes confrontés au défi de trouver les meilleurs moyens d'atteindre l'intégration à travers les distances géographiques et culturelles. Dans le cas de faire face aux défis liés au vieillissement démographique mondiale, il est soutenu que l'obtention et l'accumulation de la connaissance du contexte de la pratique locale peut être critique et productive, en particulier dans la développement d'un projet d'intervention pour des soins aux aînés. Trois types de connaissances contextuelles-à-dire, le contexte de l'expérience, le contexte de la praxis, et le contexte de la théorie - qui sont cruciales pour comprendre les situations de pratique de soins aux aînés, sont discutés, avec ses impacts possibles, sur non seulement la compréhension de la

1. Acknowledgement: This publication was supported by the National Institute on Aging and the Fogarty International Center, National Institutes of Health, through Grant Number R21 TW009263. Its contents are solely the responsibility of the authors and do not necessarily represent the official views of the NIH. 
situation des soins aux aînés dans un endroit précis, mais aussi le choix et la mise en œuvre des solutions d'intervention efficaces.

Mots-clés: connaissances contextuelles,les défis liés au vieillissement, démographique mondiale

\section{Background: A Conceptual Shift in Global Theories}

$\int$ h he later part of the $20^{\text {th }}$ century witnessed some major theoretical shifts in ways of conceptualizing human history and societal development, particularly from rather dichotomous views to integrated views. This shift is exemplified in theoretical commentaries of global political economy.

\section{Views of Dichotomy}

During the first quarter of the $20^{\text {th }}$ century, we can easily find dichotomous views of the course of development of human societies in countries such as China and Japan, where debates often focused on a dichotomy between Westernization vs. nationalism (e.g. Zhao 2000; Ishikawa 2002). Ever since, scholars, politicians, and social reformers have been divided over which approach to take at a given point of history.

Generally speaking, historians agree that in the first half of the $20^{\text {th }}$ century, throughout the two world wars, the Western view of modernization was predominant. This was based around the central notion that human societies progress through a series of universal stages of development, and a currently underdeveloped area is in a similar situation to that of the developed areas in the past. The key strategy for development in this underdeveloped area was to accelerate the speed of common paths to modernization such as urbanization, industrialization, and integration into the world market (Kendall 2007). The same period also witnessed the groundbreaking work of cultural anthropology from social thinkers, such as Franz Boas and Bronisław Malinowski, who presented a clear message with new observations that challenged the dominant view of a universal model of social and cultural progression. Their view promoted a contextualistic approach to culture and multiple possible pathways for explaining the diversity among different groups of people in different physical and social settings (Baker 2004).

The second half of the $20^{\text {th }}$ century continued to witness the persistent popularity of the modernization view along with increasingly recognized perspectives that valued local resources. In 1960s and 1970s, while modernization theory was well formulated to postulate that all societies 
progress through similar stages of development, dependency theory (Frank 1978), as a reaction to the modernization theory, began to gain popularity. Dependency theory postulated that underdeveloped areas are in a special situation, holding natural resources and large supply of cheap labor, which enables wealthy nations to perpetuate their state of dependence through a number of economic, financial, political, or even military influences. Although dependency theory focuses on the inter-relationship between wealthy and poor countries, the solution derived from this view often leads to a debate between an economic policy around the "core" (developed) countries vs. "peripheral" (underdeveloped) countries, or between a focus on strategic integration into the world market to achieve rapid economic growth vs. developing a country's domestic market and creating internal demands to aim at a slower but sustainable growth (Frank 1978). This conceptual polarity or dichotomy continued into the 1980s.

\section{Views of Integration}

From 1980s on, although the dichotomous views continued in many fields, a new conceptual shift gradually occurred, with the view of a more integrated world becoming increasingly influential in understanding how economic and social development should be organized in a global setting. The most influential is the analysis of modern world systems originated by Wallerstein $(1980,1989)$ and other scholars (e.g., Lechner 1984; Frank and Gills 1996; Barfield 1997), focusing on the worldwide conditions and mechanisms that operate as determinant forces in underdeveloped nations.

Those international mechanisms or factors, which have the greatest impact on global economic dynamic and the internal development of small countries, include new global systems of communications, world trade mechanisms, and the international financial system. The key idea, among other conceptual novelties that the world-systems theory brought, is the importance of interdependence. We are entering a world of integration and interdependence, in which not only international dynamic processes and their elements are interacting with the internal aspects of any country, but there also a range of possibilities, much broader than those based on previous dichotomous views, facing all countries. This is particularly true of those at the middle levels of development (i.e., the semiperiphery). An understanding of these possibilities and mechanisms is vital and requires a strong integration with social sciences - especially with sociology, economics, and political disciplines (Kutsche 1989). 
Derived from the world-system perspective, a more recent interdependence view of globalization recognizes the role of local context, such as culture and communication styles, as the ultimate determinant of the shape and direction of global economy and social integration. Along with the forming of the more integrated view of global development, there has been a rapid rise of interest in indigenous knowledge in the social sciences starting in 1990s, when indigenous knowledge or local wisdom, which, a few decades ago, was viewed as "inefficient, inferior, and an obstacle to development," became "an important local natural resource" that holds promise to "facilitate the development process in cost-effective, participatory, and sustainable ways (e.g., Vanek 1989; Hansen and Erbaugh 1987; Warren 1991). Indigenous knowledge and social development are no longer viewed as opposing forces, but as two, among many, different pieces of the same puzzle.

\section{Global Aging: Two Approaches towards Integration}

This trend towards a more integrated, interdependent worldview has also penetrated into the field of gerontology. Gerontology, a discipline that came to shape after WWII with primary concerns on the welfare of elders and the interface between individual elders and their surrounding social institutions, has naturally been extended to incorporate issues of global aging, as the demographics of global population aging have become increasingly recognized over the past two decades.

Global aging is an ideal example to confirm the merits of a more integrated worldview. On the one hand, many countries, both underdeveloped and developed, are facing similar trends of demographic transition due to population aging. They respond similarly in exploring solutions and strategies to cope with the looming impacts of population aging on various aspects of a society including the economy, health care, longterm care, family, and community. On the other hand, each country or each community is at its own culturo-historical place in terms of the needs for improving or maintaining their current living standards and ways of local practice, as well as the limits of that practice, in providing care, life, and value to an elder.

\section{Issue \#1}

A key challenge for a global aging agenda is how to integrate the relatively generic and often shared agendas related to social development, as well as coping with demographic changes with the indigenous knowledge and practice that is rooted in specific local culture and history. 
Through work done during the past decade, we propose two basic approaches to this possible integration. These approaches originally emerged from our experience of conducting a number of large national projects, mostly funded by the U.S. federal agencies (e.g., the Substance Abuse and Mental Health Administration, the Department of Veterans Affairs, and the Health Resources and Services Administration) to enhance mental health services for underserved ethnic minority older adults in the U.S. by developing evidence-based and culturally competent intervention services and programs. Through working with multiple service sites across the nation, we discovered two general pathways through which a culturally competent or appropriate health intervention could be developed for older adults of various cultural backgrounds. The basic idea of these two approaches has been published before in an edited volume, titled "Evidence-Based Behavioral Health Practice for Older Adults: A Guide to Implementation" (Levkoff et al. 2006).

The model is applicable to the global aging scenario partly because the tension between the evidence-based approach (a specific knowledge base to solve generic problems across different populations and settings) and cultural competence in delivering a service to a specific population in a specific setting (a more locally based challenge) is essentially the same for projects involving either minority health or global elder care. Furthermore, minority health care also needs to solve a number of issues commonly facing elder populations in the global setting (e.g., poverty, medical co-morbidity, interface between family and community resources of care). The two basic approaches are summarized below:

\section{Approach 1. From science to practice}

As a common strategy for adapting or tailoring an evidence-based intervention to a new cultural environment, this science-based approach can broadly be described in the following steps: (1) finding the best scientific evidence from available sources (e.g., published clinical guidelines, research literature of randomized controlled trials, or demonstration programs) that addresses the identified problem; (2) identifying the cultural characteristics of the target community that require modifications to the evidence-based intervention protocol and barriers that need to be addressed in order to implement an intervention; (3) adapting the evidencebased practice to the cultural and clinical service situation identified in Step 2, thus forming a new, culturally adapted protocol; (4) implementing and adhering to the adapted protocol, paying attention to changes in operational procedures and staff behaviours; and (5) confirming the effectiveness of the new intervention by collecting outcome data, including feedback from the targeted population. 


\section{Approach 2. From practice to science}

In contrast, the other approach emphasizes the importance of developing a culturally grounded or rooted intervention, rather than transplanting and adapting an established evidence-based protocol to a new cultural setting. This approach typically involves following five steps, assuming that service needs or problem areas have already been identified: (1) systematically documenting local experience and anecdotal evidence about best practices in delivering health services to ethnic minority communities; (2) summarizing the local evidence into a model by developing a service manual or protocol that may be supplemented with evidencebased principles from other sources (e.g., literature or other demonstration interventions); (3) refining the model to allow flexibility or versatility in serving different ethnic subgroups; (4) implementing the newly developed service manual and conducting a formative evaluation to ensure acceptance and feasibility of the model in the local community; and (5) conducting outcome evaluation studies to assess the effectiveness of the locally grown intervention model.

The latter, culturally grounded model is based on two main assumptions. First, service providers who are experienced in serving the local ethnic cultural community know the best way to serve that particular population. Second, the cultural characteristics of individuals and their community settings are complex, such that it might be not wise or feasible to import an intervention protocol originated in another context.

\section{Issue \#2}

This view of two pathways of integration is helpful for explaining retrospectively which path one has taken, but the key challenge is that it does not inform which pathway to apply, prospectively, in a particular situation or at a given point of research or clinical practice. We are arguing in this paper that obtaining and accumulating contextual knowledge might prove to be a productive method to assist in the planning phase of a project. This by no means suggests that it would settle any debate for those who already have a clear and often strong position toward either of these two approaches on a specific topic.

\section{Contextual Knowledge: Key to Global Aging Intervention}

We propose a solution to the challenge of decision making regarding the choice between these two basic approaches (science to practice and practice to science) to integration. The solution is based on some recent case examples and observations using ethnographic methods. In our en- 
counters and projects with our international colleagues through the past two decades, we have noticed a pattern in which choices of the types of interventions which we first thought were most appropriate changed with the contextual knowledge we obtained continuously through the process of on-going assessment of the local eldercare situation.

In collaboration with our colleagues in Thailand, for instance, a central theme of our discussions throughout the years has been what kind of intervention is needed. In the very beginning of our meetings at our first visit, leaders of Thai institutions of aging research and directors of local hospitals were present with academics from the U.S. The central challenge that we sought to address was the existence of dementia patients and the family caregivers who receive no special support from the community (Grant number: TW009263, supported by the NIH/NIA and Fogarty International Center, Principal Investigator: Chen). This knowledge about the situation was based on a combination of abstraction of the situation from other parts of the world (i.e., the universal phenomenon of dementia caregiver stress), the general information of family structure in Thailand, and the fact that there are no known community programs supporting Thai dementia caregiving families (i.e., knowledge of social structure). The conclusion we came to was to identify one of the education and counseling intervention models from the U.S. and implement it in Thailand. We thought we would follow the intervention model based on the NIA-funded REACH study (Schulz et al. 2003), which focuses on providing multi-component psycho-educational counseling to dementia caregiving families in the Thai community.

On our second round of visits, we had a chance to visit homes of persons affected with dementia and their family caregivers in rural and urban Thailand. What struck us foremost was the wide range of heterogeneity of homes not only with varying levels of living conditions, but also the variety of problems they had encountered during the course of care provision to their ill elderly family member. We also learned that many families had gone through extraordinarily difficult times in the course of the providing care to a family member who suffered from dementia or other debilitating conditions, and they typically would adapt to the situation by finding an effective solution or utilizing some coping mechanisms. Based on our understanding of the situation, we proposed to train local hospital-based nurses to be "problem-solving facilitators", similar to the role of case managers, to help individual families solve the problems they faced, in order to reduce the psychological stress that resulted from a particularly pressing elder care problem. An intervention that aimed to deliver problem-solving support, we believed, would be 
much simpler and feasible than the multi-component psycho-educational counseling offered through the REACH intervention.

On the third round of trips, we began to learn more about the community and its service and human resources. For instance, we learned that in Thailand there is a long history of organizing volunteer health workers (VHWs) to provide basic health-related liaison services and support to families residing in the same community. In many areas across provinces, especially in rural Thailand, the VHW forms a widespread network that connects the formal health care systems to individual families through activities such as regular visitation, monitoring, and referral to the health care providers (e.g., community health promotion center, different levels of hospitals). In some areas, agencies promoting eldercare have begun to select among VHWs to form a group of volunteer eldercare workers (VEWs) to provide support and care coordination to families with frail elders. Based on this learning, we changed our previous thinking (i.e., bringing a trained nurse from a local or regional hospital) to believing that the most appropriate intervention would be to strengthen the existing VEW system by adding specific components of services to what they are already providing for elders, rather than introducing a new eldercare program.

Reflecting on our experience obtained from these trips and the transformation in our view of the most appropriate intervention to recommend to promote elder care in Thailand, we see a general pattern: the more we know about the actual context of the eldercare experience, the more we tend to shift away from simply translating an evidence-based, Western-originated intervention model toward solutions based on modification, enhancement, or adjustment of an existing local service entity or system. In other words, the more contextual knowledge we have about a local situation, the more likely we will believe in the value and appropriateness of the existing local practice. The word "value" may indicate a number of things, including the ability to reach community members, to be accepted by the local community, to attain desired clinical outcome, to obtain high level of satisfaction from users of services, to financial sustainability, and a greater likelihood to be accepted by local political stakeholders thus potentially increasing the chance for being scalable beyond the original, experimenting community.

If contextual knowledge is possibly a crucial factor that affects the decisions about types of appropriate intervention projects for local settings, what do we really mean by contextual knowledge in the context of eldercare in the global aging setting? Why would contextual knowledge affect our choice of intervention? How do we improve contextual knowledge? 


\section{Types of Contextual Knowledge}

The contextual knowledge relevant to elder care in the global aging setting can be sorted into three subcategories corresponding to the following three contexts: context of experience, context of praxis, and context of theory. Such a division can be productive in designing a preliminary or planning study prior to a more focused investment of an intervention project. The three contexts as described below are, in principle, applicable to all major stakeholders of elder care (e.g., elder in need of care, family caregiver of elder, paid caregivers, health care providers in the community, government officials who help develop and execute aging policy). However, the importance and relevance of each category of contextual knowledge may differ for different stakeholders.

\section{The Context of Experience}

The context of experience is a map of one's experience, based on the information of the person's subjective experience. Like a map of geography, the map of experience highlights the key points that represent the major concerns and experiences of significance. Moreover, the map of human experience also depicts the ecological overview of the human being as a bio-social organism. For an elder or a caregiver of a frail elder, the essential information might include the following items in describing such a context of experience of a person: (a) an active needs profile that depicts the perceived, observed, or inferred needs (e.g., biological, psychological, social, cognitive needs) of an individual or a group of individuals; (b) a values profile that includes reported or observed values, preferences, and priorities of an individual; (c) resources that refer to the amount and availability of resources that exists in one's living environment (e.g., natural environment, or service system) and can potentially provide fulfillment to one's needs and values; (d) social support (tangible or symbolic, regular or occasional) that facilitates and enables needs fulfillment; (e) barriers (physical, mental, environmental, and social) to resources, fulfillment or to behaviours that are intended to lead to resources and fulfillments; and (f) trauma or significant life events in the past, as part of one's biographical narrative, that may play an important role in shaping one's needs, values, and ways of seeking resources.

The experiential context provides key information about the needs profile of a person, such as a caregiver, a family, or a community. The needs profile can be biased or false, but the bias may be reduced if interviews involve multiple informants and are administered repeatedly with careful consideration to remove bias (e.g., reporting socially desirable 
responses that are acceptable information to be given publically, distrust towards interviewer). The needs profile can also be limited by, for instance, the interviewee's perspective of caregiving and life, or the interviewee's tendency and ability to articulate life experiences, in addition to other possible bias introduced by the interviewer.

\section{The Context of Praxis}

The distinction between experience and praxis is important for understanding the nature of praxis or action as used here. First, the context of experience refers to explicit experience (e.g., feelings, life satisfaction, means of obtaining subsistence, types of support received), which is accessible in principle through the experiencer's self report. Praxis or action is about processes of one's life that are likely, though not always, to be implicit knowledge that often needs to be detected through methods other than mere self reporting. Second, the experiential context focuses on the past and present influences on the experience of one's current living situation, whereas the context of activity focuses on actions, along with the associated psychological background, that tend to carry the individual towards the future where desired changes are likely or intended to happen. As Adolfo Sánchez Vázquez (1977) wrote in his book, The Philosophy of Praxis, "Praxis... is not merely an interpretation of the world, but is also a guide to its transformation." Third, the experiential context deals more with life processes that aim toward basic and clearly identified needs, with shorter-term goals and routine structure, whereas the context of praxis often involves new directions and plans as well as exploration of possibilities of change and improvement, which is crucial for eldercare since the majority of eldercare projects involve a certain degree of improvement in care practice.

In the context of eldercare, the analysis of praxis or action, be it towards care for another person or care for oneself, is inseparable from its enabling processes, which, according to experts in studies of processes of (behavioural) change (Prochaska et al. 2008), can be described using two sets of variables: (a) individual variables such as the cognitive, affective, and evaluative factors that may affect one's intentional stance prior to the implementation of actions (e.g., the motive for a certain behaviour, intention to change, specific action plan under contemplation, habit of change or non-change); and (b) interactive variables such as conditioning, contingencies, environmental controls or requirements, and social learning/training opportunities. These two sets of variables work together in affecting some of the critical constructs such as determination and commitment of the person in relation to the course of action. 
The analysis of the context of praxis is particularly useful in understanding the possibility of positive changes that are expected to happen in a stakeholder (e.g., a family caregiver, a community health professional, or an administrator of a care organization such as a hospital). Our belief in the likelihood of a change is dependent upon our knowledge and understanding of (1) the reasons, history, and perceived reward mechanisms behind the existing, routine forms of practice, (2) the reasons behind the recent and local initiative, the poised proposal, the prepared plan, and experience of failure and success in the new practices designed towards changes and improvements.

As Jensen (2005) writes about Vygotsky's theory of action that: "The activity in itself is the context." Not only what has not been changed, the routine, but also the activity that has been directed towards changes, may all serve as either barriers or facilitators toward further changes.

\section{The Context of Theory}

The third context differs from the previous two contexts in its emphasis on explanatory frameworks for critical experience related to care. First, in both experiential and praxis contexts, we are concerned about causality, but the causal relationships are often a result of inference by the investigator based on self-report or observational data of the events. In contrast, the context of theory involves causal relationship as a theoretical assumption, a principle, a model, a value-laden belief or conclusion that is held to be true for an individual (e.g., a caregiver, or a community health worker) of interest or those around the individual. Second, while unfulfilled needs are of central concern in the experiential context, and desired goals in the praxis context, the context of theory provides a broader perspective, a worldview or a historical view, to explain the significant meaning of a particular behaviour and goal, thus justifying the conduct and often the moral significance and power of persuasion.

An adult-child caregiver, for instance, may view the task of providing care to a frail parent not only as inevitable daily chore to fulfill the basic needs of an elderly person (i.e., thinking in the context of experience), as many emerging challenges that demand new learning and problem solving (i.e., in the context of praxis), but also as an opportunity to reciprocate the kindness once received from the parent and to model an example of passing along care and kindness for future generations (i.e., viewing from the context of theory). The thought of cosmic kindness as a real and imaginary entity flowing from the act of care from one person to another, thus sustaining humanity, provides a feeling of self-affirming worthiness. Of course, not all broad perspectives are simply positive. 
Some Asian caregivers under the influence of Buddhism may think of their being a long-term caregiver as a sign of previous karma, which may become positive if it helps the caregiver accept the inevitable situation, but may also increase passivity, thus discouraging exploration and seeking new and better solution to any existing problem.

The content of the context of theory can come from various sources such as religion, family dictum, medical experts, community leaders, peer caregivers or friends, mass media, government officers, or simply abstraction of life experience and life stories. For each individual, some beliefs, abstract conclusions, or even theoretical frameworks are explicit and can be clearly articulated, while other beliefs are more implicit and may become crystallized only with the assistance of conversation, discussion, and request of agreement or disagreement over a carefully formulated statement. For some people, a theoretical framework is closely linked to their practice and other associated thoughts; for others, there might be less coherence between a strong belief and other thoughts, or between one's beliefs/values and one's action/choices.

These three contexts - the context of experience, the context of praxis, and the context of theory - provide the basic and comprehensive information about the overall context of an eldercare situation. The division is roughly consistent with Karl Popper's 'three worlds' theory that includes the objective, the subjective, and the theoretical or cultural aspects (Popper 1978).

\section{Influences of Contextual KNOWLEDGe}

All observers, initially often as an outsider to a family, a community, or a country, typically increase their contextual knowledge throughout the course of observation, but with different learning curves and patterns. Some go through a U-shaped curve; they gain contextual knowledge at a very high speed in the beginning to form initial and often simplistic impressions, and the learning slows down afterwards with persistent confusion and perplexity. Later, however, they may experience another level of breakthrough in their understanding and insight about the observed target. Others may experience a more linear incremental learning curve. In general, the increase of the contextual knowledge involves two parallel processes: the continuing accumulation — broadening and deepening - of contextual data, and reiterative construction and reconstruction of the conceptual abstraction of the subject matter.

With regard to elder care, the increase of contextual knowledge often has a clear impact on one's views of both problems of an elder care 
situation and appropriate solutions to the identified problems. In general, from the perspective of epistemology, it is no surprise that one's view of a problem or a solution has to do with one's knowledge - both previously acquired and continuously obtained. Some contextual knowledge, as described above, can directly affect one's understanding of eldercare problems. According to Piaget and other epistemologists (Landry1995), this can occur through various pathways. For instance, knowing another person's subjective experience of a problem-situation (e.g., a caregiver feeling physical pain due to carrying an elderly person) or his or her theoretical interpretation of a problem can easily affect our perspective of problems. Our observation of a discrepancy between a caregiving family's experience of a problem and their relative passivity or inaction towards solving the problem may also give us a sense of the larger problem. Moreover, as our contextual knowledge deepens, our view of problems may expand from a few initially identified points to an interconnected network or complex of problems.

Similarly, the growth of contextual knowledge is also likely to change our view of solutions to a problem in several ways. The knowledge of problems will naturally affect our view of desired end states, which will affect our mental search for means or solutions, as our minds tend to form means-ends analysis, according to cognitive scientists of problem solving processes (Newell and Simon 1972). Our contextual knowledge not only involves knowledge of the problem situation and solutions in abstract thinking, but also the knowledge of the concrete settings in which the solution may be executed, the characteristics of the person who will implement the solution, and the social and cultural environment that determines perception and interpretation of implementation of a solution: all these affect so-called contextual appropriateness of a solution or feasibility of an intervention in the implementation sciences literature (e.g., Titler 2010; Damschroder et al. 2009).

If one's contextual knowledge about a problem situation keeps growing with accumulating information about the complexity, or the interconnectedness, of the local situation, it is highly likely that one may find it increasingly difficult to identify a foreign-born solution that will fit the problem situation. Even if a foreign-born solution is identified, the amount of effort used in adapting the original solution may not be worth the effort required in the continuous need to modify the intervention over its course of development and refinement (e.g., Kitson et al. 2008). Therefore, with the growth of contextual knowledge, it will not be a surprise to see the general inclination towards, and acceptance of, a locally originated solution, or the preference of modifying or strengthening a locally grown solution to adapting or translating a foreign-born solu- 
tion, especially when the long-term concerns (e.g., community adoption, program sustainability, and broader scalability) are taken into account.

\section{General Discussion}

The central issue discussed in this paper originates from a special historical context in which the old, continuous, ever-resurgent trend of globalization meets with the new trend of global demographic aging. For centuries, globalization, driven by financial, industrial, and imperial forces, has overall been seen as a process of homogenization or "deterritorialization" of ideas, values, and standards across the boundaries of nations, often at the expense of local traditional diversity. Only recently, a contrasting trend has become more accepted, that of viewing globalization as a process towards diversification and hybridization, rather than homogenization, which not only promotes sharing and preserving local uniqueness, individuality, and identity (Osterhammel and Petersson 2005), but also increased acceptance of a pluralistic version of modernities or synthesis of cultures (Pieterse 2009). Some have even further made a distinction by calling this newer trend internationalization as a process that "recognizes national boundaries and the uniqueness of individual societies and cultures, and... urges international understanding and cooperation" (Chan 2004), rather than globalization in its traditional sense as a world-wide process that implied a "standardization across cultures that occurs as technology, migration, and education become dispersed around the globe" (McCabe 2001).

The rapid advance of information and communication technologies seems to further strengthen this new trend of globalization or internationalization as diversification. On one hand, it provides an infrastructure to accelerate transnational business and financial transactions and civil society interactions (Ferguson and Mansbach 2012). On the other hand, it also expands a space, an intellectual phase, where exchange of information and synthesis of views and knowledge across geographical and cultural distance may occur before further invested actions and transactions, thus broadening not only our consciousness of global connectivity but also the knowledge base to support a more informed decision (Sun 2008).

It is in this background that demographic aging has emerged as a global trend in that the concern of population aging and its associated burdens are occurring in many places on the planet, along with uneven preparedness for this historically unprecedented situation. Compared with developing nations, developed countries are often a few steps ahead in 
developing research and service expertise in provision of adequate eldercare, partly because they experienced demographic aging a few decades, in some cases even a century, earlier than most of developing countries. However, typically, there is a gap between the need for improving care capacity in developing countries and the solutions developed based on practice and research experience obtained from the developed nations. In some cases, reducing this gap means carefully translating and adapting the foreign grown solution to the local culture. In other cases, reducing the gap means supporting local practice and initiative by allowing it to incorporate wisdom obtained from other locales. Either case - successful adaptation of a foreign solution to a local setting or skillful cultivation of a local solution to turn it into a generalizable model or experience - often requires, or at least will benefit from, knowledge of the local context in which the elder care practice occurs.

Systematically organized contextual knowledge, of a practice in either a distant community or one's own neighborhood, can provide helpful understanding of the problem situation and therefore one's choice of interventions or solutions. Although much can be said about the advantages of holding the international perspective for its broader access and understanding of options and outcomes of various service models, the same can be true in that those with local wisdom, or deeper access and understanding of the local situation, will not be at the most advantageous position in finding the service model that are both accepted by people and sustainable in the local context. The argument of advantage of breadth versus depth will remain unsettled until further information is obtained that reveals the connotation of contextual knowledge of a locality and the meaning of a good and lasting service.

In the course of accumulating our contextual knowledge, the idea of an intervention, an intended solution to a recognized problem, can be viewed as a point or a node embedded in the network of information we call contextual knowledge. Malinowski (1935), one of the forerunners and proponents of the concept of context, wrote: “. . the meaning of a word must always be gathered, not from passive contemplation of this word, but from an analysis of its functions, with reference to the given culture." He argued against the notion that a word contains a meaning as one of its essential features, and believed that only by referring a word to its given cultural context could its meaning be properly assigned.

If we replace "word" with "intervention" in the above text, the argument seems to fit well to our current situation of global aging or global health scenario: that is, when facing the choices of intervention models for a particular setting in a new community, we must let our understanding of the meaning of an intervention go beyond that which comes from 
"passive contemplation" of the intervention protocol itself, and only by collecting and analyzing the information about possible interaction between the intervention and its implementation context, will we attain an understanding that is appropriate and sustainably beneficial to persons living in the local cultural and physical setting.

In this discussion of context as a concept, we have gone beyond the common meaning of the word context as a background (e.g., the context of a literature work or an art product) that can be used for interpretation of the work, and in a way returned to the original meaning of the Latin word contexere, as "weaving together", a state towards connection and coherence of all elements of concern.

\section{REFERENCES}

Baker, Lee D. 2004. Franz Boas out of the ivory tower. Anthropological Theory 4(1):29-51.

Barfield, T. 1997. The Dictionary of Anthropology. Oxford: Wiley-Blackwell.

Chan, W.W.Y. 2004. International cooperation in higher education: Theory and practice. Journal of Studies in International Education 8(1):32-55.

Damschroder L.J., D.C. Aron, R.E. Keith, S.R. Kirsh, J.A. Alexander, and J.C. Lowery. 2009. Fostering implementation of health services research findings into practice: A consolidated framework for advancing implementation science. Implementation Science 4:50, 1-15.

Engel, J.L. 2012. Making meaning of the American student-Spanish host family experience. Doctoral dissertation, University of South Carolina.

Ferguson, Y.H. and W. Mansbach. 2012. Globalization: The Return of Borders to a Borderless World? London: Routledge.

Frank, A.G. 1978. Dependent accumulation and underdevelopment. Monthly Review Press.

Frank, A.G. and B.K. Gills. 1996. The World System: Five Hundred Years or Five Thousand? London: Routledge.

Ishikawa, A. 2002. Modernization: Westernization vs. nationalism - A historical overview of the Japanese case. Development and Society 31(2):281-88.

Jensen, P.E. 2005. A contextual theory of learning and the learning organization. Knowledge and Process Management 12(1):53-64.

Kendall, Diana. 2007. Sociology in Our Times: The Essentials. Seventh Edition. Wadsworth Publishing.

Kitson A.L., J. Rycroft-Malone, G. Harvey, B. McCormack, K. Seers, and A. Titchen. 2008. Evaluating the successful implementation of evidence 
into practice using the PARiHS framework: Theoretical and practical challenges. Implementation Science 3(1):1-12.

Kutsche, Paul. 1989. World system theory as an integrator of the social science curriculum. Urban Anthropology and Studies of Cultural Systems and World Economic Development 18(1):129-134.

Landry, M. 1995. A note on the concept of problem. Organization Studies 16(2):315-343.

Lechner, F.J. 1984. Ethnicity and revitalization in the modern world system. Sociological Focus 17:243-256.

Malinowski, B. 1935. Coral Gardens and Their Magic: A Study of the Methods of Tilling the Soil and of Agricultural Rites in the Trobriand Islands. London: Allen \& Unwin.

McCabe, L.T. 2001. Globalization and internationalization: The impact on education abroad programs. Journal of Studies in International Education 5(2):138-145.

Newell, A. and H. A. Simon. 1972. Human Problem Solving. Englewood Cliffs, NJ: Prentice-Hall.

Osterhammel, J. and N.P. Petersson. 2005. Globalization: A Short History. Princeton, NJ: Princeton University Press

Pieterse, J.N. 2009. Globalization and Culture: Global Mélange.

Prochaska, J.O., C.A. Redding, and K.E. Evers. 2008. The transtheoretical model and stages of change. P. 105 in K. Glanz, B.K. Rimer, and K. Viswanath, eds., Health Behavior and Health Education. Fourth edition. San Francisco: Jossey-Bass.

Robertson, R. 1992. Globalization: Social Theory and Global Culture. London: Sage.

Schulz R., L. Burgio, R. Burns, C. Eisdorfer, D. Gallagher-Thompson, L.N. Gitlin, and D.F. Mahoney. 2003. Resources for enhancing Alzheimer's caregiver health (REACH): Overview, site-specific outcomes, and future directions. Gerontologist 43(4):514-20.

Sun, J. 2008. Global Connectivity and Local Transformation: A Micro Approach to Studying the Effect of Globalization on Shanghai. University Press of America.

Titler, Marita G. 2010. Translation science and context. Research and Theory of Nursing Practice 24(1):35-55.

Vázquez, A.S. (1977). The Philosophy of Praxis. The Merlin Press Ltd.

Wallerstein, I. (1980). The Modern World System II: Mercantilism and the Consolidation of the European World-Economy, 1600-1750. New York: Academic Press. 
1989. The Modern World-System III: The Second Era of Great Expansion of the Capitalist World-Economy, 1730-1840s. New York: Academic Press.

Zhao, Suiseheng. 2000. Chinese nationalism and its international orientations. Political Science Quarterly 115(1).

Hongtu Chen, Ph.D., is an Assistant Professor at Harvard Medical School, and Director of the Global Initiative on Caregiving for the Elderly at the Harvard University Asia Center. He has done extensive research related to health service intervention, especially in implementing and evaluating evidence-based, behavioural health interventions for elderly people in various clinical an cultural settings. He published widely including a co-edited book: "Beyond Experience: Implementing Evidence-Based Behavioral Health Practices for Older Adults" (2006), and special issues on "Population Aging in Thailand" (2009) and "Dementia Caregiving and Chinese Culture" (2010).

hongtuchen@hotmail.com.

Sue Levkoff, Sc.D., S.M., M.S.W., is one of world's leading experts on geriatrics and social gerontology. She currently is the Endowed Chair in Community and Social Support for SmartHOME, and Professor of Social Work, at the University of South Carolina. She has led large research projects mostly funded be U.S. federal agencies including the National Institute on Aging, the Fogarty International Center of the National Institutes of Health, and the Substance Abuse and Mental Health Administration. Levkoff current work focuses on developing innovative technologies that will enable older adults to remain at home in the community with adequate supports. She also serves as the Editor-in-Chief for the peer-reviewed journal Ageing International.

sue.e.levkoff@gmail.com.

Arthur Kleinman, M.D., is the Esther and Sidney Rabb Professor of Anthropology at Harvard University and Professor of Medical Anthropology in Global Health and Social Medicine and Professor of Psychiatry, Harvard Medical School. He currently serves as the Victor and William Fung Director of Harvard University Asia Center. Kleinman is an internationally known leader in several fields, including medical anthropology, cultural psychiatry, global health, social medicine, and medical humanities. He is a member of the Institute of Medicine of the National Academies and of the American Academy of Arts and Sciences. Kleinman is also the author of six books, coauthor of two others, coeditor of nearly 30 volumes and seven special issues of journals.

kleinman@,fas.harvard.edu. 